\title{
Pregnancy outcome after intrauterine adhesiolysis
}

\author{
Enlan Xia ${ }^{1}$, Xiaowu Huang ${ }^{1}$, Tin-Chiu $\mathrm{Li}^{1,2}$ \\ ${ }^{1}$ Hysteroscopy Center, Fuxing Hospital, Capital Medical University, Beijing 100038, China; ${ }^{2}$ Assisted Conception Unit, Department of Obstetrics \& \\ Gynaecology, Chinese University of Hong Kong, Hong Kong, China \\ Correspondence to: Tin-Chiu Li. Assisted Conception Unit, Department of Obstetrics \& Gynaecology, Chinese University of Hong Kong, Shatin, \\ Hong Kong, China. Email: tinchiu.li@gmail.com. \\ Provenance and Peer Review: This article was commissioned by the Editorial Office, Annals of Translational Medicine. The article did not undergo \\ external peer review. \\ Comment on: Feng Q, Gao B, Huang H, et al. Obstetrical outcome in the third trimester after hysteroscopic adhesiolysis. Ann Transl Med $2020 ; 8: 51$.
}

Submitted Dec 20, 2019. Accepted for publication Jan 09, 2020.

doi: $10.21037 /$ atm.2020.01.111

View this article at: http://dx.doi.org/10.21037/atm.2020.01.111

The pregnancy outcomes of women who had a history of intrauterine adhesiolysis have been previously examined by a number of investigators. A recent systematic review (1) analysed 54 studies and reported a pooled pregnancy rate of $50.7 \%$, with an early pregnancy loss rate of $17.7 \%$, ectopic pregnancy rate of $4.2 \%$, a significant increase in late pregnancy complication rates including mid-trimester loss rate of $1.5 \%$, cervical incompetence rate of $12.5 \%$ and placenta accrete syndrome of $10.1 \%$. The placenta accrete syndrome appeared more than 10 times that of the general population.

In this issue of the journal, Feng et al. (2) from Xiangya Hospital, China reported on the findings of a retrospective cohort study which included 146 women with a history of hysteroscopic adhesiolysis (HA) who had given birth in their third trimester over a 7 year period, compared with a cohort of 292 women with a negative history of HA but matched for maternal age, gravidity, parity and delivery year. They reported no significant difference between study and control cohorts in gestational weeks, birth weight, Apgar score, rates of fetal distress and neonatal intensive care unit (NICU) admissions but women with a history of HA were at a higher risk of placenta previa $(11.6 \% \mathrm{vs}$. $3.1 \%$ ), abnormally invasive placenta (AIP) (33.6\% vs. $2.7 \%$ ) and retained placenta $(42.5 \%$ vs. $8.6 \%)$. As a result, the postpartum hemorrhage (PPH) rate in the study cohort was significantly higher than that of the comparison cohort ( $8.9 \%$ versus $1.0 \%)$. Moreover, they have stratified the outcomes based on the severity of IUA and found women with severe IUA had a higher risk of complications.

Despite that women had a history of HA were at a higher risk of several of obstetric complications, the Xiangya group (2) reported in the result section that the Caesarean section delivery rate in the study cohort was only $28.8 \%$, being significantly lowered than the rate of $54.8 \%$ in the comparison cohort. In the discussion section, the authors on the other hand referred to a higher Caesarean section rate in the study cohort. It is unclear if the Caesarean section rate in the study cohort was indeed lower than that of the comparison group. If so, it would be a rather unusual observation on its own.

The retrospective nature and the special design of the study by Feng et al. (2019) which included only subjects who had a delivery in the third trimester meant that it was not possible to draw conclusion on mid-trimester complications such as mid-trimester loss, cervical incompetence \& and preterm delivery before 28 weeks gestation.

Nevertheless, the study by Feng et al. (2) of a sufficiently large cohort with a matched comparison group did confirm the findings of a recent systematic analysis [Guo et al., (1)] that women who conceived after surgical treatment of IUA have increased obstetric complications and should be proactively managed. At the very least, women should have the possible risks explained to them and be offered additional monitoring in the antenatal period, with delivery conducted in a hospital setting by an experienced obstetric team.

How can the complications reported by Feng et al. (2) be reduced? A most important strategy is primary prevention, that is, prevention of the occurrence of intrauterine adhesion in the first instance. It is now understood that intrauterine adhesion occurs as a result of damage to the endometrium, especially in the gravid state. The avoidance of uterine 
curettage in women who have miscarriage is an important measure; medical management or expectant measures are often effective. The same consideration applies to women seeking termination of pregnancy, as medical management has been shown to be highly effective. If indeed evacuation of the uterus is required, it ought to be performed gently, care being taken to avoid unnecessary or excessive curettage. Performing the procedure under ultrasound guidance will let the surgeon know when the cavity is empty and so remove the need to perform check curettage. If evacuation of the uterus is required after a miscarriage, the placement of hyaluronic acid gel in the uterine cavity after the evacuation procedure has been found in a randomised control trial to reduce adhesion formation (3). It remains to be seen whether other alternatives such as a second look hysteroscopy in an outpatient setting or the use of other adhesion barrier such as an intrauterine balloon are also effective primary prevention measures. The use of adhesion prevention agents should also be considered when resecting multiple submucosal fibroids as it carries a higher risk of intrauterine adhesion formation when compared with resection of a solitary fibroid.

Once intra-uterine adhesions have occurred, they may cause a number of symptoms such as menstrual irregularity especially oligomenorrhoea or amenorrhoea, infertility, recurrent miscarriage and a number of late pregnancy complications as reported in the study by Feng et al. (2). Treatment of intrauterine adhesions requires hysteroscopic surgery to remove the adhesions under direct vision with the use of scissors (cold steel) or diathermy needle or loop (hot wire). A major challenge of HA is the high rate of recurrence especially in cases with severe disease. Secondary preventive measures of recurrence of adhesions include early second look hysteroscopy, the use of adhesion barriers such as an intrauterine contraceptive device or intrauterine balloon, hyaluronic acid gel, amnion graft and adjuvant estrogen therapy to promote regeneration of the damaged endometrium. A recently published randomised control trial showed that the use of intermittent intrauterine balloon therapy, 2 and 6 weeks after intrauterine adhesiolysis, significantly reduced the incidence of adhesion reformation when compared with a control group (4).

Obstetricians providing care for women should take heed of the observations regarding the increase in risk of placental complications. Careful examination of the placenta by ultrasonography and doppler study ought to be planned in the third trimester to look for features of placenta accrete which if present should help in the planning of delivery.

\section{Acknowledgments}

Funding: None.

\section{Footnote}

Conflicts of Interest: The authors have no conflicts of interest to declare.

Ethical Statement: The authors are accountable for all aspects of the work in ensuring that questions related to the accuracy or integrity of any part of the work are appropriately investigated and resolved.

Open Access Statement: This is an Open Access article distributed in accordance with the Creative Commons Attribution-NonCommercial-NoDerivs 4.0 International License (CC BY-NC-ND 4.0), which permits the noncommercial replication and distribution of the article with the strict proviso that no changes or edits are made and the original work is properly cited (including links to both the formal publication through the relevant DOI and the license). See: https://creativecommons.org/licenses/by-nc-nd/4.0/.

\section{References}

1. Guo EJ, Chung JPW, Poon LCY, et al. Reproductive outcomes after surgical treatment of asherman syndrome: A systematic review. Best Pract Res Clin Obstet Gynaecol 2019;59:98-114.

2. Feng Q, Gao B, Huang H, et al. Obstetrical outcome in the third trimester after hysteroscopic adhesiolysis. Ann Transl Med 2020;8:51.

3. Hooker AB, de Leeuw R, van de Ven PM, et al. Prevalence of intrauterine adhesions after the application of hyaluronic acid gel after dilatation and curettage in women with at least one previous curettage: short-term outcomes of a multicenter, prospective randomized controlled trial. Fertil Steril 2017;107:1223-31.e3.

4. Shi X, Saravelos SH, Zhou Q, et al. Prevention of postoperative adhesion reformation by intermittent intrauterine balloon therapy: a randomised controlled trial. BJOG 2019;126:1259-66.

Cite this article as: Xia E, Huang X, Li TC. Pregnancy outcome after intrauterine adhesiolysis. Ann Transl Med 2020;8(5):154. doi: 10.21037/atm.2020.01.111 